\title{
Validação do Roteiro de Entrevista de Práticas Educativas para Professores (RE-HSE-Pr)
}

\author{
Alessandra Turini Bolsoni-Silva ${ }^{1}$ \\ Universidade Estadual Paulista, Bauru-SP, Brasil \\ José Egídio Barbosa Oliveira, Sonia Regina Loureiro \\ Universidade de São Paulo, Ribeirão Preto-SP, Brasil
}

\section{RESUMO}

No contexto escolar, habilidades sociais de professores e crianças, assim como problemas de comportamento concorrem para o desenvolvimento social e a aprendizagem dos alunos, verificando-se carência de instrumento que avalie de modo integrado tais variáveis. Com tal propósito, objetiva-se apresentar o instrumento RE-HSE-Pr e sua validação. O RE-HSE-Pr foi elaborado na mesma racional de instrumento já aferido, tendo os pais como fonte de informação, e sua apresentação após validação é de nove perguntas guias, organizadas em três eixos: Comunicação, Afeto e Estabelecimento de limites. Participaram da amostra os professores de 283 crianças pré-escolares e escolares que responderam ao RE-HSE-Pr e ao Teacher's Report Form. Procedeu-se, conforme as metodologias técnicas, as avaliações das validades de construto e discriminante e fidedignidade. Identificaram-se dois fatores (total positivo; total negativo), com alfa adequado e as análises discriminantes diferenciaram casos de não casos, meninos de meninas, pré-escolares de escolares. Os indicadores psicométricos foram satisfatórios.

Palavras-chave: habilidades sociais educativas; práticas educativas; problemas de comportamento; habilidades sociais; escola.

ABSTRACT - Validity of the Interview Script of Educational Practices for Teachers (RE-HSE-Pr)

In the school context, social skills in teachers and children, as well as child behavioral problems, contribute to the social development and learning of the students, however, there is no instrument that evaluates these variables in an integrated way. Therefore, the aim was to investigate the validation of the Interview Script of Educational Practices for Teachers (RE-HSE-Pr) instrument. The RE-HSEPr was developed in a similar way to other pre-existing instruments in which parents are the information source. After validation, it had 9 guide-questions organized on three information axes: Communication, Affection and Establishment of Limits. Participants were teachers of 283 pre-school and school age children, who answered both the RE-HSE-Pr and Teacher's Report Form (TRF). Evaluations of the construct and discriminant validity and reliability were performed, according to technical procedures. Two factors with adequate alpha (positive total; negative total) were identified, and discriminant analyses discriminated cases from non-cases; boys from girls and pre-school children from school children. Psychometric indicators were satisfactory.

Keywords: educational social skills; educational practices; behavior problems; social skills; school.

RESUMEN - Validación de la Guía de Entrevistas de Prácticas Educativas para Profesores (RE-HSE-Pr)

En el contexto escolar, las habilidades sociales de profesores y niños, así como problemas de conducta, concurren para el desarrollo social y el aprendizaje de los alumnos, comprobando carencia de un instrumento que evalúe de modo integrado tales variables. Con este propósito, se pretende presentar el instrumento RE-HSE-Pr y su validación. El RE-HSE-Pr fue elaborado de igual modo que el instrumento ya evaluado teniendo los padres como fuente de información. Su presentación, después de la validación, es de 9 preguntas guías, organizadas en tres ejes: Comunicación, Afecto y Establecimiento de límites. Participaron de la muestra profesores de 283 niños preescolar y escolares que respondieron al RE-HSE-Pr y al Teacher's Report Form (TRF). Se procedió según los procedimientos técnicos, las evaluaciones de la validez de constructo y discriminante y fiabilidad. Se identificó dos factores (total positivo, total negativo), con alfa adecuado y los análisis discriminantes diferenciaron casos de no casos, niños de niñas, preescolares de escolares. Los indicadores psicométricos fueron satisfactorios.

Palabras-clave: habilidades sociales educativas; prácticas educativas; problemas de conducta; habilidades sociales; escuela.

No contexto escolar, as habilidades sociais educativas de professores, assim como as habilidades sociais e os problemas de comportamento das crianças, concorrem para o desenvolvimento social e a aprendizagem dos alunos. Considera-se ser relevante mapear as habilidades sociais infantis importantes para a aprendizagem e socialização, assim como identificar as habilidades sociais educativas dos professores na interação com as crianças, uma

${ }^{1}$ Endereço para correspondência: Faculdade de Ciências - UNESP, Campus de Bauru. Avenida Eng. Luiz Edmundo Carrijo Coube, 14-01, Vargem Limpa, 17033-360, Bauru, SP. Tel.: (14) 3103-6087, ramal 9760. E-mail: bolsoni@fc.unesp.br

Apoio: CNPq. Bolsa de Produtividade em Pesquisa. 
vez que são comportamentos positivos deles que podem prevenir o surgimento de problemas de comportamento (Barreto, Freitas, \& Del Prette, 2011; Bolsoni-Silva \& Mariano, 2014; Bolsoni-Silva, Mariano, Loureiro, \& Bonaccorsi, 2013).

Habilidades sociais infantis e problemas de comportamento são condutas correlacionadas inversamente (Barreto et al., 2011; Berry \& O'Connor, 2010; BolsoniSilva, \& Loureiro, 2011; Kettler, Elliott, Davies, \& Griffi, 2011; Kim, Doh, Hong, \& Choi, 2011; Reynolds, Sander, \& Irvin, 2010), sendo verificada maior frequência de habilidades sociais em crianças sem problemas de comportamento, tanto pré-escolares como escolares, segundo relatos de pais e de professores (Bolsoni-Silva \& Loureiro, 2016). Considera-se que identificar, precocemente, problemas de comportamento e habilidades sociais é importante para promover o desenvolvimento e evitar o surgimento ou agravamento dos problemas de comportamento (Rauch \& Lanphear, 2012; Flett \& Hewitt, 2013; Samarakkody, Fernando, McClure, Perera, \& De Silva, 2012).

O termo "habilidades sociais" (HS) pode ser definido como comportamentos emitidos pelo indivíduo diante das demandas de uma situação interpessoal (Del Prette \& Del Prette, 1999), de forma a resolver problemas imediatos e evitar problemas futuros. Os problemas de comportamento, segundo Achenbach e Rescorla (2001), podem ser denominados de externalizantes (desafio, impulsividade, agressão, hiperatividade, desobediência) ou de internalizantes (ansiedade, retraimento, depressão).

A escola, segundo Wiley, Siperstein, Forness e Brigham (2010), é um contexto importante para a prevenção de problemas externalizantes e internalizantes. No contexto escolar, a maior fonte de queixa dos professores é a indisciplina, relacionada a problemas externalizantes dos alunos (Guerra et al., 2015), os quais são frequentemente encaminhamos para serviços de atendimento, sobretudo os meninos (Landale, Lanza, Hillemeier, \& Oropesa, 2013; Samarakkod et al., 2012). Guerra et al. (2015) analisaram as queixas de professores ao encaminharem crianças para um centro de atendimento educacional especializado e constataram que, em 92 encaminhamentos escolares, $69 \%$ das queixas eram de problemas externalizantes e apenas 16 dessas crianças tinham diagnóstico para o atendimento em educação especial (Sindrome de Down, Transtorno do Espectro Autista, Hipotireodismo, Paralisia Cerebral, Síndrome de Dand Walker e Hidrocefalia), sendo, portanto, a grande maioria das crianças encaminhadas sem diagnóstico. Os meninos são comumente referidos como os que apresentam mais problemas de comportamento externalizantes (Cosentino-Rocha \& Linhares, 2013; Landale et al., 2013; Samarakkody et al., 2012), enquanto as meninas tendem a ter repertório mais elaborado de habilidades sociais (Leman \& Bjornberg, 2010) mas, em contrapartida, apresentam mais problemas internalizantes (Cosentino-Rocha \& Linhares, 2013).

Achados quanto à influência da escolaridade no repertório infantil não são consensuais. Estudos sugerem que as crianças escolares parecem ser mais habilidosas que as pré-escolares, tanto na visão dos professores (Bolsoni-Silva, Marturano, \& Freiria, 2010) como na das mães (Berry \& O'Connor, 2010). Bolsoni-Silva et al. (2016) identificaram que os meninos e os escolares tiveram mais problemas de comportamento (internalizantes, externalizantes e comorbidades), além de maior dificuldade acadêmica. Nesse sentido, o comportamento da criança tem influência de múltiplas variáveis e dentre elas está o manejo do professor, que pode ser compreendido do ponto de vista das habilidades sociais educativas.

Para Del Prette e Del Prette (2001), as habilidades sociais educativas (HSE) são voltadas intencionalmente para a promoção da aprendizagem e do desenvolvimento, seja em situação formal, seja informal. Del Prette e Del Prette (2008), a partir de uma revisão de literatura da área, propõem quatro categorias amplas para as HSE: (a) estabelecer contextos interativos potencialmente educativos; (b) transmitir ou expor conteúdos sobre habilidades sociais; (c) estabelecer limites e disciplina; (d) monitorar positivamente. Bolsoni-Silva, Loureiro e Marturano (2016), com base em um amplo estudo empírico com pais, validaram o Roteiro de Habilidades Sociais Educativas Parentais (RE-HSE-Pais) e propuseram que habilidades sociais educativas podem ser classificadas em três grandes eixos, a saber, Comunicação, Afeto e Limites, sendo importantes para a interação com os filhos, podendo promover as habilidades sociais ou favorecer o surgimento/manutenção de problemas de comportamento.

O RE-HSE-P é um instrumento que avalia a interação estabelecida entre pais e filhos elaborado a partir da literatura de habilidades sociais e de práticas educativas e que permite descrever e pontuar práticas educativas positivas e negativas, bem como comportamentos infantis de habilidades sociais e de problemas de comportamento. Na literatura, é o único instrumento que avalia concomitantemente práticas educativas e comportamentos infantis de maneira interacional, em um total de 70 itens. O instrumento foi reconhecido como um teste psicológico pelo Conselho Federal de Psicologia e está publicado enquanto manual em Bolsoni-Silva et al. (2016) e como artigo empírico em Bolsoni-Silva e Loureiro (2010). Os estudos psicométricos encontraram manutenção dos achados em análises teste-reteste, discriminação de crianças com e sem problemas de comportamento tanto para os indicadores de práticas como de comportamentos infantis. $\mathrm{O}$ alfa obtido foi de 0,846 e a análise fatorial encontrou dois fatores, que juntos, explicam $60,775 \%$ da variância, denominados de total positivo e de total negativo, sendo 
que o primeiro agrupa práticas positivas e habilidades sociais infantis e o segundo agrega práticas negativas e problemas de comportamento.

Os construtos presentes no RE-HSE-P, em estudos diversos, aparecem como variáveis importantes também nas interações professor-aluno no que se refere às práticas utilizadas e aos comportamentos infantis. Como exemplos, pode-se citar: comunicação (Fonseca, 2012; Uysal \& Ergenekon, 2010), estabelecimento de regras (Mariano \& Bolsoni-Silva, 2016), oferecimento de suporte (BolsoniSilva \& Mariano, 2014), manejo positivo (Ribeiro, 2010) e do afeto (Baker, Grant, \& Morlock, 2008; Ribeiro, 2010). Alguns estudos avaliaram habilidades sociais do professor, a partir do IHS-Del Prette, validado para uso com universitários (Maia, Soares, \& Victoria, 2010; Reis, Prata, \& Soares, 2012; Soares \& Mello, 2010), constatando que os professores têm bom repertório de habilidades sociais, mas os que atuam no ensino infantil apresentavam escores mais elevados (Maia et al., 2009).

Analisando-se a literatura da área, constata-se, assim, que muitos são os comportamentos que o professor precisa ter para maximizar o desenvolvimento das crianças e até onde pode alcançar essa revisão não foi identificado um instrumento validado que avaliasse uma diversidade de comportamentos dos professores na interação com os alunos. Nesse sentido, tomando por referência o RE-HSE-P, propôs-se adaptá-lo para aplicar com professores de forma a descrever como ocorrem as interações sociais no contexto escolar. Foram realizados estudos exploratórios com uma versão preliminar do RE-HSEPr, considerando os três eixos de HSE (Bolsoni-Silva \& Mariano, 2014; Mariano \& Bolsoni-Silva, 2016). Nessas pesquisas, pôde-se notar que a qualidade da comunicação e afeto, bem como das estratégias para estabelecer limites influenciavam o comportamento de crianças pré-escolares e escolares. No entanto, o instrumento em sua versão original era muito extenso (155 itens), o que dificultava a coleta e a adesão de participantes, o que motivou ajustá-lo. Assim, verificando-se carência de um instrumento que avalie de modo integrado as variáveis habilidades sociais educativas e práticas negativas de professores, assim como as habilidades sociais e os problemas de comportamento das crianças no contexto escolar, é que surge o presente estudo que se propõe a apresentar o instrumento RE-HSE-Pr e testar as suas propriedades psicométricas quanto à validade e fidedignidade.

\section{Método}

\section{Participantes}

Participaram do estudo os professores de 283 crianças (166 meninos, 117 meninas) sendo 141 mulheres e oito homens. Apenas 12 desses professores tinham menos de dois anos de experiência, variando de 1 a 30 anos $\left(M=13,52 ; D P=8,19^{2}\right)$. Dessas crianças, são 122 (59 meninos e 63 meninas) de escolas municipais infantis
(EMEI) e 161 (107 meninos e 54 meninas) de escolas municipais de ensino fundamental (EMEF). Os pré-escolares tinham de 1 a 6 anos de idade $(M=3,86 ; D P=1,05)$ e os escolares têm de 5 a 12 anos (média $=8,45 ; D P=1,62$ ).

\section{Percurso Amostral}

$\mathrm{Na}$ cidade do interior paulista onde foi conduzida a coleta de dados, há 16 escolas municipais de ensino fundamental (EMEFs), com um total de 287 professores, do primeiro ao quinto ano, sendo em média 17 professores por escola. Já as escolas municipais infantis (EMEIs) contam com 38 unidades, tendo 554 professores, em média, com 14 professores por escola. Na presente pesquisa, foram colhidos dados junto a todas as 16 EMEFs e em 16, das 38 EMEIs. As EMEIs que foram convidadas a participar estavam na mesma região demográfica da EMEF correspondente e, quando havia recusa, outra EMEI que atendesse a esse critério foi convidada. Desse modo, considerando o número de escolas da cidade, foram colhidos dados em $100 \%$ das escolas EMEFs e em $42 \%$ das EMEIs da Rede Municipal de Ensino.

Nas EMEFs, participaram da pesquisa 95 professores, tendo, portanto, 192 recusas. Já nas EMEIs, 52 de 224 professores convidados responderam aos instrumentos, havendo 172 recusas. Com essa amostra de professores, era previsto que fossem avaliadas 298 crianças, mas foram de fato avaliadas 283 , uma vez que alguns professores não indicaram uma criança com problemas e outra sem problemas de comportamento, pois quando não identificavam ambas as crianças em sua sala avaliaram apenas.

Quanto às características dos professores, a idade variou de 22 a 64 anos $(M=38,74 ; D P=9,38)$, o tempo de serviço relatado foi de 1 a 30 anos $(M=13,52 ; D P=8,19)$ e $96 \%$ concluíram curso superior. Dos respondentes, $88 \%$ relataram conhecer bem ou muito bem as crianças avaliadas por eles. O número de alunos por sala variou de $13 \mathrm{a}$ $35(M=24,67 ; D P=5,59)$. O número de escolas em que o professor trabalhava variou de 1 a 3 (52,3\% uma escola; $42 \%$ duas escolas e 3,2\% três escolas). Quanto ao número de períodos em que trabalhava, a maioria relatou trabalhar dois períodos $(65,7 \%)$. O número de turmas em que o professor lecionava concentrou-se em uma $(40,6 \%)$ ou duas turmas $(43,6 \%)$. A renda familiar dos professores variou de três a acima de seis salários mínimos: três salários $=7,4 \%$; quatro salários $=17 \%$; cinco salários $=14,1 \%$; seis salários $=16,3 \%$; acima de seis salários $=37,1 \% ; 8,1 \%$ dos respondentes não informaram a renda.

\section{Instrumentos}

TRF - Teachers Report Form (Inventário de Comportamento da Infância e Adolescência, Achenbach, \& Rescorla, 2001), respondido pelos professores com a finalidade de identificação de crianças com e sem problemas de comportamento, o que serviu de suporte para a escolha dos itens a serem mantidos no instrumento em 
validação. O TRF pode ser aplicado em pré-escolares, a partir de um ano e meio e em escolares. Ele avalia a frequência de respostas indicativas de problemas de comportamento. Os resultados são organizados em problemas internalizantes, externalizantes e totais, sendo uma avaliação mundialmente utilizada, considerada padrão ouro para mensurar problemas de comportamento, adaptado culturalmente para o português (Duarte \& Bordin, 2000) e encontra-se em fase de validação.

RE-HSE-Pr - Roteiro de Habilidades Sociais Educativas Parentais (adaptado de Bolsoni-Silva et al., 2016). Avalia a interação professor-aluno. Foi elaborado na mesma racional do instrumento RE-HSE-P (Bolsoni-Silva et al., 2016; Bolsoni-Silva \& Loureiro, 2010) já aferido, o qual tem os pais como fonte de informação, como apresentado previamente.

$\mathrm{Na}$ sua versão original, o RE-HSE-Pr contava com 155 itens a partir de 14 perguntas-guia que foram elaboradas considerando o estudo de Bolsoni-Silva et al. (2016), sendo que, após a validação, permaneceram no instrumento nove perguntas-guias e 80 categorias (exemplo dessas categorias estão descritas a seguir). Estudos prévios (por exemplo, Mariano \& Bolsoni-Silva, 2016; Mariano \& Bolsoni-Silva, prelo) mostraram a viabilidade em avaliar as práticas educativas do professor e os comportamentos infantis com o instrumento de 14 perguntas-guia, mas dada a sua extensão, o tempo de aplicação era alto e dificultava a adesão dos participantes. Nesse sentido, para sua readequação e redução de itens, foram adotados alguns critérios para a manutenção dos itens, a saber, aqueles que de forma explícita caracterizavam ter ou não ter problemas de comportamento; procurou-se manter um número equivalente de itens positivos e negativos para professores e alunos, considerando para tal a presença de correlações fortes ou moderadas, e ainda preservaram-se os itens respondidos como presentes por pelo menos $5 \%$ da amostra. Na sua versão final, o instrumento passou a contar com nove perguntas-guia e 80 itens, os quais são organizados em três grandes categorias: Comunicação, Afeto e Limites. Tais itens são codificados em Habilidades Sociais Educativas (HSE-Pr), Práticas Negativas (PR NEG), Contexto (CON), Problemas de Comportamento (PROBL) e Habilidades Sociais (HS).

As nove perguntas guias do RE-HSE-Pr são: 1. Você conversa com seu aluno(a)? 2. Você faz perguntas a seu aluno(a)? 3. Você expressa seus sentimentos negativos a seu aluno(a)? 4. Você expressa suas opiniões a seu aluno(a)? 5. Você estabelece limites para seu aluno(a)? 6. Seu aluno(a) faz coisas de que você gosta? 7. Seu aluno(a) faz coisas de que você NÃO gosta? 8. Você demonstra carinho ao seu aluno(a)? 9. Acontece de você fazer algo em relação a seu aluno(a) e sentir como errado? Para cada uma delas, o entrevistador anota ( ) SIM ou ( ) NÃO. Se a resposta for SIM, seguem as demais questões que investigam as situações (contextos) em que o comportamento ocorre, a forma como o professor emite a habilidade social educativa e como o aluno se comporta. A título de exemplo para a questão sobre estabelecimento de limites, as perguntas propostas são: (a) Por que é importante na sua opinião estabelecer limites? (b) Em que situações você costuma estabelecer limites? (c) O que você faz para estabelecer limites? (e) Como seu aluno(a) reage ao limite? Ou o que ele faz nestas ocasiões?

Após a entrevista ser completada e que, por sugestão deverá ser gravada, o pesquisador/clínico deverá preencher um protocolo para registrar as respostas de acordo com categorias estabelecidas no processo de validação. Essa codificação em detalhe está presente no manual do teste que foi aprovado pelo Conselho Federal de Psicologia (Bolsoni-Silva, Marturano \& Loureiro, 2018). A título de exemplo, o sistema de codificação para estabelecimento de limites é:

5. Você estabelece limites para seu aluno (a)?

$\operatorname{Sim}(\quad)$ Não ( )

a) Por quê?

40. CONT: Para preservar a saúde dos alunos

41. CONT: Para orientar concepções de certo e errado

número de itens ( )

número de itens ( )

Outro:

b) Em que situações você costuma estabelecer limites?
42. CONT: concepções de certo e errado
43. CONT: situações acadêmicas
44. CONT: situações diversas
45. CONT: situações de lazer
46. PROBL: externalizante

número de itens $(\quad$ )
número de itens $(\quad)$
número de itens $(\quad$ )
número de itens $(\quad$ )
número de itens $(\quad$ )

Outro: 
5. Você estabelece limites para seu aluno (a)?

$\operatorname{Sim}(\quad)$ Não ( )

c) O que você faz para estabelecer limites?

47. HSE-Pr: Conversa sobre concepções de certo e errado.

número de itens ( )

48. HSE-Pr: Comunicando-se.

número de itens ( )

49. PR NEG: Não habilidoso ativo.

número de itens ( )

Outro:

d) Como seu aluno(a) reage ao limite? Ou o que ele faz nestas ocasiões?

50. HS: Disponibilidade social e cooperação.

número de itens ( )

51. HS: Expressando sentimento e enfrentamento.

52. PROBL: externalizante

número de itens ( )

53. PROBL: internalizante

número de itens ( )

número de itens ( )

Então, o entrevistador, após codificar, terá um conjunto de 80 respostas, sendo os itens alocados em categorias de Habilidades Sociais Educativas (HSE-Pr), Práticas Negativas (PR NEG), Variáveis De Contexto (CONT), Habilidades Sociais Infantis (HS) e Problemas de Comportamento (PROBL externalizante e internalizante), as quais são agrupadas em Total Positivo e Total Negativo, de acordo com a validação de construto.

\section{Procedimentos de Coleta de Dados}

Após a aprovação da Secretaria de Educação Infantil de uma cidade de porte médio do interior paulista, foram contatadas as 16 Escolas de Educação Infantil (EMEI) e 16 de Ensino Fundamental (EMEF), sendo apresentados os objetivos do presente trabalho para a diretora, coordenadora pedagógica e professores.

Todos os professores de EMEIs e de EMEFs que aceitaram colaborar com o estudo foram solicitados a indicarem duas crianças, da sala sob sua responsabilidade, uma que considerassem ter problemas de comportamento e outra sem problemas de comportamento. Os professores que aceitaram participar, responderam aos instrumentos em uma sessão de aplicação, de aproximadamente uma hora, após o consentimento explícito por parte dos pais/responsáveis.

\section{Procedimentos de Tratamento e Análise de Dados}

Para a validação do instrumento RE-HSE-Pr, preliminarmente foram conduzidos estudos de fidedignidade (alfa) e estrutura interna do instrumento (análise fatorial exploratória). Na sequência, foram realizados os estudos para testar as validades discriminantes. Tais análises estão de acordo com o recomendado por Pasquali, (2000).

Em um primeiro momento, foi conduzida a análise fatorial exploratória considerando os 80 itens do instrumento, o que se mostrou inviável, dada a identificação de 31 fatores. Dessa forma, optou-se por agrupar os itens em categorias - método utilizado na validação do RE-HSE-P (Bolsoni-Silva, Loureiro, \& Marturano, 2016), após consenso entre três juízes independentes. $O$ próximo passo foi conduzir uma nova rodada da análise fatorial, considerando as seguintes categorias mais amplas: Habilidades Sociais Educativas (HSE-Pr); Práticas Negativas (PR NEG); Contexto (CON); Problemas de Comportamento (PROBL) e Habilidades Sociais (HS). Verificou-se que a análise fatorial com as cinco categorias explicou 67,782 da variância, tendo sido extraídos dois fatores: Fator 1, composto por práticas negativas e problemas de comportamento; e Fator 2, composto por contexto, habilidades sociais educativas do professor e habilidades sociais das crianças. Analisando-se a contribuição das categorias para a constituição dos fatores, optou-se por retirar a categoria Contexto, por ter uma natureza diversa das demais, sendo que a explicação com quatro categorias passou a ser de 78,192 da variância, conforme Tabela 1. Manteve-se a análise fatorial com as categorias Habilidades Sociais Educativas (HSE-Pr); Práticas Negativas (PR NEG); Problemas de Comportamento (PROBL) e Habilidades Sociais (HS). Utilizou-se o método de extração de análise do componente principal, com rotação varimax. $\mathrm{O}$ alfa foi calculado considerando todos os itens do RE-HSE-Pr, inclusive os de contexto e considerando os itens que ficaram após a análise fatorial.

As análises discriminantes com teste $t$ de Student foram conduzidas, considerando às seguintes comparações de grupos: (a) ter indicativo de problema de comportamento x não ter tais indicativos; (b) meninos x meninas; (c) pré-escolares x escolares.

\section{Resultados}

A seção de resultados inclui os estudos de fidedignidade (alfa) e a validade de construto (análise fatorial) que são descritos na Tabela 1. As Tabelas 2, 3 e 4 apresentam, 
respectivamente, os achados das validades discriminantes quanto à presença ou não de problemas de comportamento, para as variáveis Sexo e Escolaridade das crianças.

De acordo com a Tabela 1, o instrumento possui dois fatores, sendo o primeiro deles denominado de Total Negativo que agrupa os problemas de comportamento e as práticas negativas do professor. No segundo fator, denominado Total Positivo, encontram-se as habilidades sociais infantis e as práticas positivas do professor (HSEPr). Juntos, os dois fatores explicam 78,192\% da variância, com KMO e Barlett adequados. O alfa é de 0,649 para os 80 itens e de 0,666 para os 64 itens.

Tabela 1

Alfa dos Itens e Matriz Fatorial das Categorias Comportamentais do RE-HSE-Pr, com Análise dos Componentes Principais e Rotação Varimax

\begin{tabular}{|c|c|c|}
\hline Categorias do RE-HSE-Pr & $\begin{array}{c}\text { Fator } 1 \\
\text { (27 itens) }\end{array}$ & $\begin{array}{c}\text { Fator } 2 \\
\text { (37 itens) }\end{array}$ \\
\hline Problemas de comportamento & 0,916 & \\
\hline Habilidades sociais & & 0,821 \\
\hline HSE-Pr & & 0,856 \\
\hline Prática negativa & 0,862 & \\
\hline \% da variância explicada pelo fator & 43,000 & 35,192 \\
\hline \% da variância total acumulada & & 78,192 \\
\hline \multicolumn{3}{|l|}{$\mathrm{KMO}=0,494$} \\
\hline \multicolumn{3}{|l|}{ Barlett: $p=0,000$} \\
\hline \multicolumn{3}{|l|}{ Alfa $(80 \text { itens })^{*}=0,649$} \\
\hline Alfa (64 itens) $=0,666$ & & \\
\hline
\end{tabular}

Nota. ${ }^{*}$ Incluindo a categoria contexto

Tabela 2

Validade Discriminante: Médias, Desvios Padrão e Resultados do Teste t Student (2-Tailed) na Comparação entre os Grupos com e sem Indicativos de Problema de Comportamento em pelo menos uma das Escalas do TRF

\begin{tabular}{|c|c|c|c|c|}
\hline \multirow{3}{*}{ Categorias do RE-HSE-Pr } & \multicolumn{2}{|c|}{ Média (DP) } & \multirow{3}{*}{$t$} & \multirow{3}{*}{$p$} \\
\hline & \multicolumn{2}{|c|}{$\begin{array}{c}\text { Indicadores de problemas } \\
\text { de comportamento }\end{array}$} & & \\
\hline & $\begin{array}{c}\text { Presente* } \\
(n=142)\end{array}$ & $\begin{array}{c}\text { Ausente } \\
(n=141)\end{array}$ & & \\
\hline HSE-Pr & $11,82(3,75)$ & $11,10(3,46)$ & 1,689 & 0,570 \\
\hline Habilidades sociais & $7,51(3,56)$ & $8,61(3,41)$ & $-2,643$ & 0,009 \\
\hline Contexto & $5,99(2,98)$ & $6,61(2,89)$ & $-1,768$ & 0,078 \\
\hline Práticas negativas & $2,92(2,82)$ & $0,91(1,62)$ & 7,359 & 0,000 \\
\hline Problemas de comportamento & $11,77(6,92)$ & $3,89(4,87)$ & 11,073 & 0,000 \\
\hline Total positivo & $19,34(6,18)$ & $19,74(5,83)$ & $-0,569$ & 0,570 \\
\hline Total negativo & $14,69(8,74)$ & $4,79(5,89)$ & 11,173 & 0,000 \\
\hline
\end{tabular}

A Tabela 2 apresenta diferenças quanto às comparações entre os grupos clínico e não clínico para problemas de comportamento, sendo que o grupo clínico tem menos habilidades e mais problemas de comportamento, e os professores usam mais práticas negativas. Tais achados configuram na maior presença de Total Negativo para o grupo clínico na comparação com o não clínico.

As comparações entre meninos e meninas e entre pré-escolares e escolares são apresentadas na Tabela 3. Verificaram-se diferenças para categorias dos dois fatores, sendo que, no Fator 1, todas discriminaram, indicando que os meninos apresentam, segundo relato dos professores, mais problemas de comportamento e que usa-se com eles mais práticas negativas, o que culmina no alto escore para o total negativo. Quanto às categorias da interação positiva, apenas as habilidades sociais infantis discriminaram os grupos, sendo mais frequentes para as meninas.

Nas comparações entre pré-escolares e escolares, foram identificadas diferenças para as habilidades sociais educativas, variáveis de contexto e total positivo, todas com maiores médias para a pré-escola em comparação com o período escolar. As práticas negativas e problemas de comportamento não diferenciaram os escolares. 
Tabela 3

Validade Discriminante: Médias, Desvios Padrão e Resultados do Teste t Student (2-Tailed) na Comparação entre Meninos e Meninas e na Comparação entre Pré-Escolares e Escolares

\begin{tabular}{|c|c|c|c|c|}
\hline \multirow[b]{2}{*}{ Categorias do RE-HSE-Pr } & \multicolumn{2}{|c|}{ Média (DP) } & \multirow[b]{2}{*}{$t$} & \multirow[b]{2}{*}{$p$} \\
\hline & $\begin{array}{c}\text { meninos } \\
(n=167)\end{array}$ & $\begin{array}{c}\text { meninas } \\
(n=116)\end{array}$ & & \\
\hline HSE-Pr & $11,65(3,78)$ & $11,20(3,37)$ & 1,046 & 0,296 \\
\hline Habilidades sociais & $7,62(3,33)$ & $8,70(3,71)$ & $-2,514$ & 0,013 \\
\hline Contexto & $6,07(3,12)$ & $6,64(2,66)$ & $-1,658$ & 0,099 \\
\hline Práticas negativas & $2,63(2,84)$ & $0,89(1,43)$ & 6,118 & 0,000 \\
\hline Problemas de comportamento & $10,39(7,29)$ & $4,17(5,11)$ & 7,931 & 0,000 \\
\hline Total positivo & $19,29(5,98)$ & $19,90(6,04)$ & $-0,829$ & 0,408 \\
\hline Total negativo & $13,02(9,20)$ & $5,06(6,02)$ & 8,181 & 0,000 \\
\hline \multirow[b]{2}{*}{ Categorias do RE-HSE-Pr } & \multicolumn{2}{|c|}{ Média (DP) } & & \\
\hline & $\begin{array}{c}\text { pré-escolares } \\
(n=122)\end{array}$ & $\begin{array}{c}\text { escolares } \\
(n=161)\end{array}$ & $t$ & $p$ \\
\hline HSE-Pr & $12,22(3,76)$ & $10,89(3,42)$ & 3,074 & 0,002 \\
\hline Habilidades sociais & $8,45(4,03)$ & $7,76(3,07)$ & 1,628 & 0,105 \\
\hline Contexto & $7,24(3,10)$ & $5,59(2,62)$ & 4,732 & 0,000 \\
\hline Práticas negativas & $1,63(2,20)$ & $2,14(2,71)$ & $-1,732$ & 0,084 \\
\hline Problemas de comportamento & $7,43(6,79)$ & $8,16(7,44)$ & $-0,858$ & 0,391 \\
\hline Total positivo & $20,67(6,56)$ & $18,68(5,41)$ & 2,793 & 0,006 \\
\hline Total negativo & $9,06(8,37)$ & $10,29(9,35)$ & $-1,168$ & 0,244 \\
\hline
\end{tabular}

\section{Discussão}

O RE-HSE-Pr, após sua readequação e redução, configurou-se em dois fatores, um direcionado à interações sociais professor-aluno positivas e outro relacionado às interações negativas. Tais fatores também foram identificados no RE-HSE-P, na versão aplicada a pais/cuidadores (Bolsoni-Silva et al., 2016), indicando associações entre práticas negativas e problemas de comportamento e habilidades sociais educativas dos educadores e habilidades sociais infantis. Esses achados corroboram pesquisas prévias conduzidas com pais (Bolsoni-Silva et al., 2016; Sabbag \& Bolsoni-Silva, 2011) e com professores (Mariano \& Bolsoni-Silva, 2016) e também com ampla literatura da área de habilidades sociais (Barreto et al., 2011; Berry \& O'Connor, 2010; Kettler at al., 2011; Kim et al., 2011; Reynolds et al., 2010), sendo reiterado que as habilidades sociais e problemas de comportamentos são inversamente proporcionais. Nas análises discriminantes, foi identificado que as habilidades sociais infantis, os problemas de comportamento e as práticas negativas diferenciaram crianças com e sem problemas de comportamento e, ainda, diferenciaram meninos de meninas. Portanto, o REHSE-Pr foi capaz de discriminar as crianças, avaliadas por professores, como tendo ou não problemas de comportamento e também de acordo com o sexo.

Nas comparações entre meninos e meninas, constatou-se que meninos apresentaram escores mais baixos de habilidades sociais (Leman \& Bjornberg, 2010) e escores mais altos de comportamentos problema
(Cosentino-Rocha \& Linhares, 2013; Landale et al., 2013; Samarakkody et al., 2012). As comparações demonstraram que as práticas educativas são diferenciadas para meninos e meninas, sendo que os professores usam mais práticas negativas (Silver, Measelle, Armstrong, \& Essex, 2010) com os meninos, o que pode explicar, ao menos em parte, a permanência de mais problemas de comportamento em meninos e mais habilidades sociais em meninas. Baker (2006) observou maior frequência de relacionamentos de conflito e raiva mútua entre professores e alunos do sexo masculino e ainda que o grupo de meninas apresentava mais relações de proximidade com professores e menos relações de conflito, indicando boa adaptação ao ambiente escolar, considerando os comportamentos de estudar, seguir regras e acompanhar a rotina da escola. Para Doumen et al. (2008), esse fenômeno de conflito ocorre devido à influência mútua de comportamentos agressivos nas interações entre professores e crianças, podendo intensificar os padrões de comportamentos-problema no decorrer da escolarização.

Nota-se que as habilidades sociais educativas do professor não diferenciaram crianças com e sem problemas de comportamento diferentemente do encontrado em Bolsoni-Silva e Mariano (2014), possivelmente por terem estudado uma amostra apenas de escolares e em menor número. De todo modo, os professores pareceram ser habilidosos ao conversarem para estabelecerem limites e também por serem afetuosos com seus alunos, o que parece concordar com pesquisas que identificaram bom repertório de habilidades sociais por parte dos 
professores (Maia et al., 2009; Reis et al., 2012; Soares \& Mello, 2010). Bolsoni-Silva e Mariano (2014) notaram, nesse estudo também que a qualidade da comunicação foi diferente para os grupos com e sem problemas de comportamento, sendo para os primeiros mais relacionados ao estabelecimento de limites e, ao segundo sobre temas diversos e acadêmicos que incluíam assuntos de interesse dos alunos. Para futuras pesquisas, pode ser importante verificar, junto aos professores, quais situações de conflitos eles não conseguem aplicar as estratégias positivas de educação, ainda que o façam frequentemente, de forma a diminuir o uso de práticas negativas na interação com os alunos. Desse modo, parece que a qualidade da utilização de práticas positivas faz diferença, uma vez que conversar sobre diversidade de assuntos e situações e ser mais afetivo promove habilidades sociais e reduz problemas de comportamento.

A comparação das práticas educativas de educadores e comportamentos infantis em dois momentos de diferentes escolarizações (pré-escolares e escolares) demonstrou que professores relataram ser mais habilidosos, com maior variedade de contextos na interação com crianças da pré-escola, possivelmente por ser um ambiente mais lúdico quando comparado à idade escolar, o qual possivelmente implica em maior exigência e limites para a criança e maior demanda de aprendizagem formal. Tal dado é semelhante ao relatado no estudo de Maia et al. (2009) que constatou mais escores de habilidades sociais em professores de pré-escolares. Os achados também corroboram a pesquisa de Baker (2006) que identificou uma tendência à diminuição de relacionamentos positivos entre professores e alunos ao longo da escolarização. Crianças pré-escolares e escolares não se diferenciaram quanto às habilidades sociais e problemas de comportamento, concordando com Mariano e BolsoniSilva (2016) e discordando da literatura que indica os escolares como mais habilidosos (Berry \& $\mathrm{O}^{\prime}$ Connor, 2010; Konold, Jamison, Stanton-Chapman, \& RimmKaufman, 2010) ou com mais problemas de comportamento (Bolsoni-Silva et al., 2016), o que pode ser resultante de peculiaridades das amostras estudadas.

Considerando o conjunto de achados da presente pesquisa, verifica-se, em concordância com a literatura, um conjunto de habilidades dos professores que poderão melhorar as habilidades sociais infantis e reduzir problemas de comportamento, tais como a competência em conversar (Fonseca, 2012; Uysal \& Ergenekon, 2010), estabelecer regras/limites (Mariano \& Bolsoni-Silva, 2016; Mariano \& Bolsoni-Silva, 2018), oferecer suporte ao aluno ao conversar sobre assuntos acadêmicos (BolsoniSilva \& Mariano, 2014), valorizar bons comportamentos (Fonseca, 2012), manejar positivamente conflitos (Ribeiro, 2010) e ser afetuoso (Baker et al., 2008; Ribeiro, 2010). Assim, de um modo integrado, com foco na interação professor aluno, foi possível identificar habilidades educativas dos professores, práticas negativas, problemas de comportamento e habilidades sociais infantis. Destaca-se que os fatores identificados no RE-HSE-P (Bolsoni-Silva \& Loureiro, 2010) foram os também obtidos com o RE-HSE-Pr, indicando que a adaptação para o contexto escolar para o estudo de práticas e comportamentos infantis traz singularidades, mas também semelhanças com as interações pais e filhos.

As habilidades sociais pareceram atuar como fator de proteção ao desenvolvimento infantil, previnindo o surgimento e/ou manutenção de problemas de comportamentos (Reynolds et al., 2010). Considera-se que as ações de prevenção são necessárias para evitar o surgimento de problemas de comportamento (Rauch \& Lanphear, 2012; Samarakkody et al., 2012) e para tal é importante identificá-los precocemente (Flett \& Hewitt, 2013), sendo a escola um ambiente que pode prevenir problemas externalizantes e internalizantes (Wiley et al., 2010) e os professores podem ser bons informantes.

\section{Considerações Finais}

Os estudos psicométricos do RE-HSE-Pr mostraram indicadores satisfatórios e, nesse sentido, a elaboração e validação de um instrumento que avalia a interação professor-aluno mostrou-se relevante por permitir a identificação sistemática de padrões comportamentais que podem favorecer ou dificultar o desenvolvimento infantil. Assim, o RE-HSE-Pr, considerando a avaliação simultânea de habilidades sociais educativas, práticas educativas negativas, queixas de problemas de comportamento, habilidades sociais infantis e variáveis contextuais, contempla uma lacuna importante da literatura, podendo ter aplicabilidade no contexto escolar ao permitir a identificação de recursos e de dificuldades das crianças e dos professores, o que poderá favorecer a condução e a avaliação da efetividade de intervenções, seja com os professores, seja com os alunos. Considerando as limitações do estudo, sugere-se que estudos futuros avaliem amostras de diferentes origens escolares e que associem medidas de observação às medidas de relato, além de conduzirem análises psicométricas adicionais, como análise fatorial confirmatória e curvas Roc.

\section{Referências}

Achenbach, T. M., \& Rescorla, L. A. (2001). Manual for the ASEBA School-Age Forms E Profiles. Burlington, VT: University of Vermont, Research Center for Children, Youth \& Families. 
Baker, J. A. (2006). Contributions of teacher-child relationships to positive school adjustment during elementary school. Journal of School Psychology, 44(3), 211-229. doi: 10.1016/j.jsp.2006.02.002

Baker, J. A., Grant, S., \& Morlock, L. (2008). The teacher-student relationship as a developmental context for children with internalizing or externalizing Behavior. School Psychology Quarterly, 23(1), 3-15. doi: 10.1037/1045-3830.23.1.3

Barreto, S. O., Freitas, L. C., \& Del Prette, Z. A. P. (2011). Habilidades sociais na comorbidade entre dificuldade de aprendizagem e problemas de comportamento: Uma avaliação multimodal. Psico (Porto Alegre), 42(4), 503-510. Recuperado de http://revistaseletronicas.pucrs.br/ ojs/index.php/revistapsico/article/view/7593/7457

Berry, D., \& O'Connor, E. (2010). Behavioral risk, teacher-child relationships, and social skill development across middle childhood: A child-by-environment analysis of change. Journal of Applied Developmental Psychology, 31(1), 1-14. doi: 10.1016/j.appdev.2009.05.001

Bolsoni-Silva, A. T., \& Loureiro S. R. (2011). Práticas educativas parentais e repertório comportamental infantil: Comparando crianças diferenciadas pelo comportamento. Paidéia, 21(48), 61-71. doi: 10.1590/S0103-863X2011000100008

Bolsoni-Silva, A. T., \& Loureiro, S. R. (2016). Simultaneous assessement of social skills and behavior problems: Education and gender. Estudos de Psicologia (Campinas), 33(3), 453-464. doi: 10.1590/1982-02752016000300009

Bolsoni-Silva, A. T., \& Loureiro, S. R. (2010). Validação do Roteiro de Entrevista de Habilidades Sociais Educativas Parentais (REHSE-P). Avaliação Psicológica, 9(1), 63-75, 2010. Recuperado de http://pepsic.bvsalud.org/scielo.php?script=sci_arttext\&pid $=$ S1677-04712010000100008

Bolsoni-Silva, A. T., Loureiro, S. R., \& Marturano, E. M. (2016). Roteiro de Entrevista de Habilidades Sociais Educativas Parentais - Manual Técnico - RE-HSE-P. São Paulo: Cetepp/Hogrefe.

Bolsoni-Silva, A. T., \& Mariano, M. L. (2014). Práticas educativas de professores e comportamentos infantis, na transição ao primeiro ano do Ensino Fundamental. Estudos e Pesquisas em Psicologia (Online), 14(3), 814-833. doi: 10.12957/epp.2014.13912

Bolsoni-Silva, A. T., Mariano, M. L., Loureiro, S. R., \& Bonaccorsi, C. (2013). Contexto escolar: Práticas educativas do professor, problemas de comportamento e habilidades sociais infantis. Revista Psicologia Escolar e Edcuacional, 17(2), 259-269. doi: 10.1590/S141385572013000200008

Bolsoni-Silva, A. T., Marturano, E. M., \& Freiria, L. R. B. (2010). Indicativos de Problemas de Comportamento e de Habilidades Sociais em Crianças: Um Estudo Longitudinal. Psicologia: Reflexão e Crítica, 23(3), 506-515. doi: 10.1590/S0102-79722010000300011

Bolsoni-Silva, A. T., Marturano, E. M., \& Loureiro, S. R., \& (2018). Roteiro de Entrevista de Habilidades Sociais Educativas de Professores - Manual Técnico - RE-HSE-Pr. São Paulo: Cetepp/Hogrefe.

Bolsoni-Silva, A. T., Silveira, A. M. S., Cunha, E. V., Silva, L. L., \& Orti, N. P. (2016). Problemas de comportamento e funcionamento adaptativo no Teacher's Report Form (TRF): comparações por gênero e escolaridade. Gerais: Revista Interinstitucional de Psicologia, 9(1), 141-155. Recuperado de http://pepsic.bvsalud.org/pdf/gerais/v9n1/v9n1a11.pdf

Cosentino-Rocha, L., \& Linhares, M. B. M (2013). Temperamento de crianças e diferenças de gênero. Paidéia, 23(54), 63-72. doi: 10.1590/1982-43272354201308

Del Prette, Z. A. P., \& Del Prette, A. (1999). Psicologia das habilidades sociais: Terapia e educação. Petrópolis, RJ: Vozes.

Del Prette, Z. A. P., \& Del Prette, A. (2001). Psicologia das relações interpessoais: Vivências para o trabalho em grupo. Petrópolis, RJ: Vozes.

Del Prette, Z. A. P., \& Del Prette, A. (2008). Um sistema de categorias de habilidades sociais educativas. Paideia, 18(41), 517-530. doi: 10.1590/S0103-863X2008000300008

Doumen, S., Verschueren, K., Buyse, E., Germeijs, V., Luyckx, K., \& Soenens, B. (2008). Reciprocal relations between teacher-child conflict and aggressive behavior in kindergarten: A three-wave longitudinal study. Journal of Clinical Child and Adolescent Psychology, 37(3), 588599. doi: 10.1080/15374410802148079

Duarte, C. S., \& Bordin, I. A. S. (2000). Instrumentos de avaliação. Revista Brasileira de Psiquiatria, 22(Suppl. 2), 55-58. doi: 10.1590/S151644462000000600015

Flett, G. L., \& Hewitt, P. L. (2013). Disguised distress in children and adolescents "flying under the radar": Why psychological problems are underestimated and how schools must respond. Canadian Journal of School Psychology, 28(1), 12-27. doi: 10.1177/0829573512468845

Fonseca, B. C. R. (2012). Práticas educativas de genitores e professoras e repertório comportamental de crianças do ensino fundamental: Estudo de caso (Dissertação de Mestrado). Universidade Estadual Paulista. Faculdade de Ciências, Bauru, SP.

Guerra, B. T., Rovaris, J. A., Mariano, M., Guidugli, P. M., Rosanti, S., \& Bolsoni-Silva, A. T., (2015). Análise das queixas da rede municipal encaminhadas para a Educação Especial. Revista Quadrimestral da Associação Brasileira de Psicologia Escolar e Educacional, 19(2), $321-328$. doi: 10.1590/2175-3539/2015/0192841

Kettler, R. J., Elliott, S. N., Davies, M., \& Griffin, P. (2011). Testing a multi-stage screening system: Predicting performance on Australia's national achievement test using teachers' ratings of academic and social behaviors. School Psychology International, 33(1), 93-111. doi: 10.1177/0143034311403036

Kim, M., Doh, H., Hong, J. S., \& Choi, M. (2011). Social skills training and parent education programs for aggressive preschoolers and their parents in South Korea. Children and Youth Services Review, 33(6), 838-845. doi: 10.1016/j.childyouth.2010.12.001

Konold, T. R., Jamison, K. R., Stanton-Chapman, T. L., \& Rimm-Kaufman, S. E. (2010). Relationships among informant based measures of social skills and student achievement: A Longitudinal Examination of differential effects by sex. Applied Developmental Science, 14(1), 18-34. doi: 10.1080/10888690903510307

Landale, N. S., Lanza, S. T., Hillemeier, M., \& Oropesa, R. S. (2013). Health and development among Mexican. Black and White preschool children: An integrative approach using latent class analysis. Demographic Research Volume, 28(44), 1302-1338. doi: 10.4054/ DemRes.2013.28.44

Leman, P. J., \& Bjornberg, M. (2010). Conversation, development, and gender: A study of changes in children's concepts of punishment. Child Development, 81(3), 958-971. doi: 10.1111/j.1467-8624.2010.01445.x.

Maia, R. C. C., Soares, A. B., \& Victoria, M. S. (2010). Um estudo com professores da educação infantil e do ensino fundamental sobre suas habilidades sociais e inteligência geral. Estudos e Pesquisas em Psicologia, 9(2), 464-479. Recuperado de http://pepsic.bvsalud.org/pdf/epp/ v9n2/v9n2a13.pdf

Mariano, M., \& Bolsoni-Silva, A. T. (2016). Comparações entre práticas educativas de professores, habilidades sociais e problemas de comportamento de alunos. Estudos e Pesquisas em Psicologia, 16(1), 140-160. Recuperado de http://pepsic.bvsalud.org/scielo. php?script $=$ sci_arttext\&pid $=$ S1808-42812016000100009 
Mariano, M., \& Bolsoni-Silva, A. T. (prelo). Social interactions between teachers and students: a study of associations and predictions. Paidéia, 28(e2816), 1-10 doi: 10.1590/1982-4327e2816

Rauch, S. A., \& Lanphear, B. P. (2012). Prevention of disability in children: Elevating the role of environment. The Future of Children, 22(1), 193-217.

Reis, V. T. C., Prata, M. A. R., Soares, A. B. (2012). Habilidades sociais e afetividade no contexto escolar: Perspectivas envolvendo professores e ensino-aprendizagem. Psicologia Argumento, 30(69), 347-357. Recuperado de https://periodicos.pucpr.br/index.php/ psicologiaargumento/article/view/23290/22363

Ribeiro, M. L. (2010). A afetividade na relação educativa. Estudos de Psicologia (Campinas), 27(3), 403-412. doi: 10.1590/S0103166X2010000300012

Reynolds, M. R., Sander, J. B., \& Irvin, M. J. (2010). Latent curve modeling of internalizing behaviors and interpersonal skills through elementary school. School Psychology Quarterly, 25(4), 189-201.

Sabbag, G. M., \& Bolsoni-Silva, A. T. (2011). A relação das Habilidades Sociais Educativas e das práticas educativas maternas com os problemas de comportamento em adolescentes. Estudos e Pesquisas em Psicologia (UERJ. Impresso), 11(2), 423-441. Recuperado de http:// www.revispsi.uerj.br/v11n2/artigos/html/v11n2a04.html

Samarakkody, D., Fernando, D., McClure, R., Perera, H., \& DeSilva, H. (2012). Prevalence of externalizing behavior problems in Sri Lankan preschool children: Birth, childhood and sociodemografic risk factors. Social Psychiatry Epidemiologic, 47(5), 757-762. doi: 10.1007/s00127-011-0377-5

Silver, R. B., Measelle, J. R., Armstrong, J. M., \& Essex, M. J. (2010). The impact of parents, child care providers, teachers, and peers on early externalizing trajectories. Journal of School Psychology, 48(6), 555-583. doi: 10.1016/j.jsp.2010.08.003

Soares, A. B., Mello, \& T. V. S. (2010). Habilidades sociais entre professores e não professores. Revista Brasileira de Terapias Cognitivas, 5(2), 15-27. Recuperado de http://pepsic.bvsalud.org/pdf/rbtc/v5n2/v5n2a04.pdf

Uysal, A., \& Ergenekon, Y. (2010). Social skills instruction carried out by teachers working at private special education institutions in Turkey. Education and training in autism and developmental disabilities, 45(3), 459-466. Recuperado de http://tiny.cc/37kg5y

Wiley, A., Siperstein, G., Forness, S., \& Brigham, F. (2010). School context and the problem behavior and social skills of students with emotional disturbance. Journal of Child \& Family Studies, 19(4), 451-461. doi: 10.1007/s10826-009-9316-4

\section{Sobre os autores}

Alessandra Turini Bolsoni-Silva é psicóloga, professora do Departamento de Psicologia da Faculdade de Ciências - Universidade Estadual Paulista (UNESP), Bauru, e orientadora junto ao Programa de Pós-Graduação em Psicologia do Desenvolvimento e Aprendizagem.

José Egídio Barbosa Oliveira é psicólogo, doutor pela Universidade do Porto, Portugal e, atualmente faz pós-doutorado na Faculdade de Filosofia, Ciências e Letras de Ribeirão Preto - Universidade de São Paulo.

Sonia Regina Loureiro é psicóloga, professora do Departamento de Neurociências e Ciências do Comportamento da Faculdade de Medicina de Ribeirão Preto - Universidade de São Paulo (FMRP-USP) e orientadora junto aos Programas de Pós-Graduação em Psicologia FFCLRP - USP e Saúde Mental - FMRP - USP. 